\title{
MRI Classification of Subtalar Joint Osteoarthritis Using a Novel Scoring System
}

\author{
Razi Zaidi' ${ }^{1}$, Rikin Hargunani'2, Michele Calleja ${ }^{2}$, Jonathan Foley³, Andy Goldberg ${ }^{4,5,6}$ (i) \\ ${ }^{1}$ University Hospital Lewisham, Lewisham High St, London, UK \\ ${ }^{2}$ Royal National Orthopaedic Hospital, Brockley Hill, Stanmore, UK \\ ${ }^{3}$ Lothian NHS, Waverleygate, Waterloo Place, Edinburgh, UK \\ ${ }^{4}$ UCL Institute of Orthopaedics \& Musculoskeletal Science, London, UK \\ ${ }^{5}$ MSK Lab, Imperial College, London, UK \\ ${ }^{6}$ London Ankle \& Arthritis Centre, Wellington Hospital, London, UK \\ Email: razi.zaidi@nhs.net
}

How to cite this paper: Zaidi, R., Hargunani, R., Calleja, M., Foley, J. and Goldberg, A. (2020) MRI Classification of Subtalar Joint Osteoarthritis Using a Novel Scoring System. Open Journal of Radiology, 10, 69-78

https://doi.org/10.4236/ojrad.2020.102008

Received: February 16, 2020

Accepted: June 7, 2020

Published: June 10, 2020

Copyright $\odot 2020$ by author(s) and Scientific Research Publishing Inc. This work is licensed under the Creative Commons Attribution International License (CC BY 4.0).

http://creativecommons.org/licenses/by/4.0/

(c) (i) Open Access

\begin{abstract}
Background: Plain radiography usual method to detect degeneration in the subtalar and talonavicluar joints. MRI is a better way to fully characterise non-ossified structures, such as articular cartilage, marrow tissue and synovial fluid and therefore detect changes of arthritis. The motivation behind this study was to develop a quantitative way to score arthritic changes to the subtalar and talonavicular joints using MRI. The developed system will then be used as a research tool and in the close assessment and monitoring of patients with hindfoot degenerative disease. Methods: The MRI scans of thirty consecutive subjects with foot and ankle pain were retrospectively evaluated. Images were interpreted independently by three musculoskeletal radiologists in order to determine intra-observer reliability as well as the inter-observer reliability of the score. Five features of osteoarthritis were scored in the Subtalar joint and the Talonavicular joint. These were cartilage morphology, subarticular marrow, subarticular cyst, marginal osteophytes and synovitis. Results: For the 30 MRI scans the mean score for the Subtalar joint ranged from 11.7 to 14.4 and for the Talonavicular joint ranged from 3.7 to 5.6. The inter-observer correlation for the Subtalar joint between the three readers ranged between 0.53 and 0.83 for the individual features but overall was excellent at 0.76 . For the Talonavicular joint the total correlation was good at 0.67 . The inter-observer ICC for the total score was 0.75 which showed excellent agreement between the three readers. The total intra-observer correlation was excellent. Conclusions: The current work has shown excellent reliability for the scoring system. It will be a useful tool to diagnose and monitor disease progression of the Subtalar and Talonavicluar joints.
\end{abstract}




\section{Keywords}

Magnetic Resonance Imaging, Subtalar, Talonavicular, Osteoarthritis, Cartilage

\section{Introduction}

The sublatar joint is formed by the articulation of the talus and the calcaneus or talocalcaneal joint (TCJ). The calcaneus has three facets: posterior, middle and anterior that articulate with the talus (Figure 1). The anterior facet is also continuous with the navicular articulation called the talonavicular joint (TNJ) (Figure 2). The TNJ and calcaneocuboid joints (CCJ) are also called the transverse tarsal or Chopart joint (articulation tarsi transversa). Together, the TCJ, TNJ and CCJ are collectively known as the triple joints as they closely interact with each other. The subtalar joint proper however should be defined from a functional point of view as
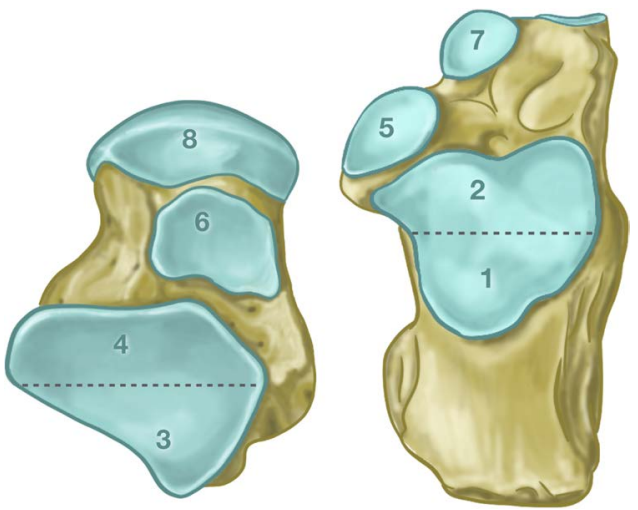

Figure 1. Illustration of the inferior articulating surfaces of the talus (left image) and superior articulating surfaces of the calcaneum (right image), showing eight regions in which the subtalar joint was scored. Surfaces $8,6,4 \& 3$ articulate with 7, 5, 2 \& 1 respectively at the Talocalcaneal Joint (TCJ). The posterior facet is comprised of 1, 2, $3 \& 4$ and the middle facet of surfaces $5 \& 6$, and the anterior facet of surfaces $7 \& 8$.

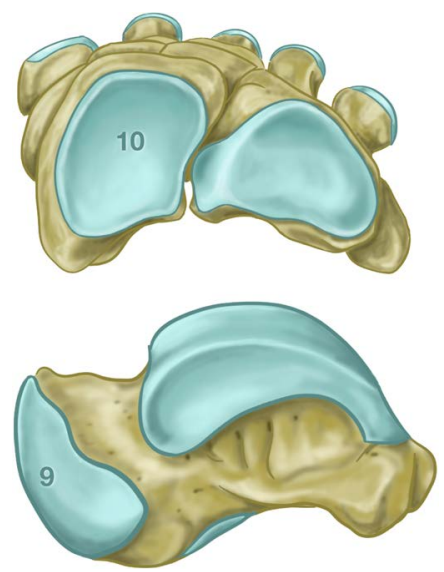

Figure 2. Illustration of the articulating surfaces of the navicular (left image) and anterior talus (right image) showing the 2 regions in which the talo-navicular joint was scored. Surfaces $9 \& 10$ articulate at the talonavicular joint. 
the osteoligamentous complex formed by the talus, the calcaneus, and the navicular, and the corresponding ligamentous complex.

Plain radiography is the usual method to detect degeneration in these joints, however since plain films do not permit full characterisation of non-ossified structures, such as articular cartilage, marrow tissue and synovial fluid any early change of degeneration can be missed. In addition, the bones and joints have complicated shapes making diagnosis of joint space narrowing or degeneration difficult to diagnose on plain standing radiographs without special views.

MRI scoring systems for hip and knee denegation have been used in research and clinical practice for some time [1] [2]. To our knowledge, no MRI scoring system for the Subtalar joint and talonavicular joint exist. The aim of this study was to develop a quantitative way to score arthritic changes to the subtalar joints (TCJ and TNJ) using MRI that is usable, repeatable and reliable. The study formed part of the feasibility phase for an NIHR HTA funded clinical trial (ISRCTN60672307) [3].

\section{Methods}

\subsection{Subjects}

Thirty ankle Magnetic Resonance Imaging (MRI) examinations performed at the Royal National Orthopaedic Hospital (RNOH), Stanmore, UK were retrospectively evaluated. Fifteen patients were known to have subtalar (TCJ or TNJ) OA. Fifteen controls were included with no known subtalar pathology. Exclusion criteria included previous foot and ankle surgery and patients under the age of 18 years.

\subsection{MRI Protocol}

MRI images were obtained using 1.5-T MRI (Achieva, Philips, The Netherlands) with an extremity surface coil. A standard protocol was used in all patients, consisting of four sequences: sagittal proton density-weighted (PDW) fast spin echo (FSE) [TR/TE 2000-3000/20, slice thickness $=3 \mathrm{~mm}$ ], sagittal short tau inversion recovery (STIR) [TR/TE 3000-4000/60, slice thickness $=3 \mathrm{~mm}$ ], axial PD FSE [TR/TE 3000-4000/30, slice thickness $=3 \mathrm{~mm}$ ] and coronal PD FSE fat suppressed images [TR/TE 3000-4000/25, slice thickness $=4 \mathrm{~mm}$ ].

\subsection{Image Analysis}

MR images were interpreted independently by a musculoskeletal radiology fellow (JF) and two specialised, fellowship-trained musculoskeletal radiologists (RH and MC). In order to determine intra-observer reliability as well as the inter-observer reliability two of the readers independently scored the studies twice, more than 14 days apart.

\subsection{Development of Score}

Based on a review of the literature and expert opinion obtained using a modified 
Delphi Method [4] (five features were identified that reflect the process of osteoarthritis (OA) in the subtalar joint (Table 1) [5] [6] [7] [8].

The first of these features was cartilage morphology which was scored 0-3, representing the spectrum of normal cartilage to diffuse full thickness loss (Figure 3). Subarticular marrow was the second feature to be assessed, with abnormality defined as poorly marginated areas of increased signal intensity in the normally fatty subarticular marrow on fat-suppressed or STIR images. This was scored 0 - 3 and covered a spectrum from normal to sclerosis and bone marrow oedema (Figure 4). The third feature was subarticular cysts scored 0 to 2, with 0 meaning none and 2 meaning multiple cysts (Figure 5). These were identified

Table 1. Table of the features assessed in the scoring system.

\begin{tabular}{|c|c|c|}
\hline \multicolumn{3}{|c|}{ Features scored with Stanmore scoring system } \\
\hline Feature & Range & Scoring \\
\hline Cartilage & $0-3$ & $\begin{array}{l}\text { 0-Normal thickness and signal } \\
\text { 1-Partial thickness loss } \\
\text { 2-Focal full thickness loss } \\
\text { 3-Diffuse full thickness loss }\end{array}$ \\
\hline Subarticular marrow abnormality & $0-3$ & $\begin{array}{l}0 \text { - None } \\
1 \text {-Oedema } \\
\text { 2-Oedema and sclerosis } \\
\text { 3-Sclerosis }\end{array}$ \\
\hline Subarticular cysts & $0-2$ & $\begin{array}{l}\text { 0-None } \\
\text { 1-Present } \\
\text { 2-Multiple cysts }\end{array}$ \\
\hline Marginal osteophytes & $0-1$ & $\begin{array}{l}0 \text {-None } \\
1 \text {-Present }\end{array}$ \\
\hline Synovitis & $0-1$ & $\begin{array}{l}0 \text {-Normal } \\
1 \text {-Present }\end{array}$ \\
\hline
\end{tabular}

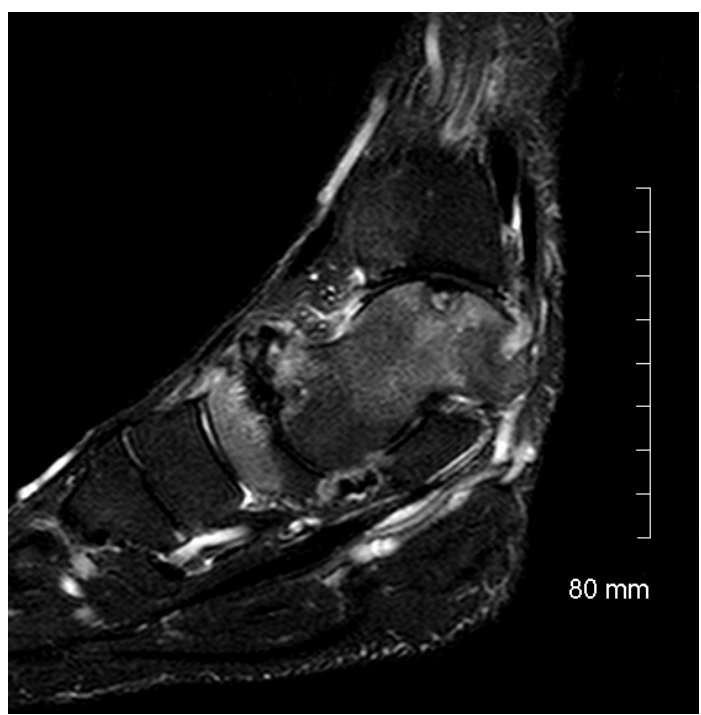

Figure 3. Sagittal MR image demonstrating diffuse areas of full thickness cartilage loss along the talonavicular joint. 


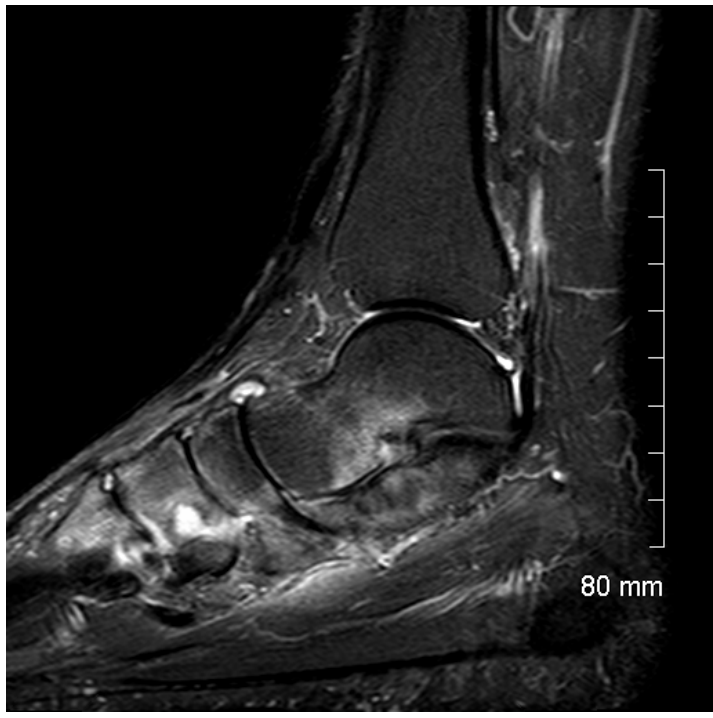

Figure 4. Sagittal STIR MR image of the subtalar joint showing subarticular bone marrow oedema.

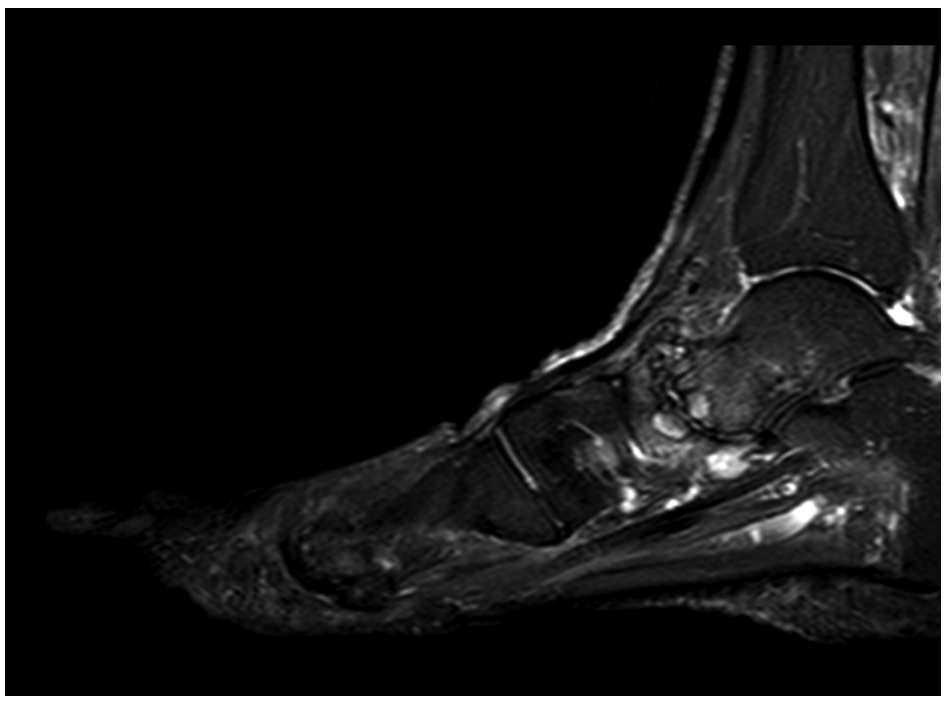

Figure 5. Sagittal STIR MR image showing multiple cysts along the talo-navicular joint margins.

typically as ovoid foci of fluid signal intensity in the subarticular bone. Osteophytes along joint margins were scored, graded from 0 (none) or 1 (present), representing the fourth feature assessed (Figure 6). The fifth graded feature was synovitis which was graded 0 (absent) or 1 (present) (Figure 7).

The TCJ was scored in eight different regions (Figure 1) and TNJ in two (Figure 2). Five features were scored in each region (Table 1). The maximum score for both regions combined was 100 with higher scores representing more severe osteoarthritis.

\subsection{Statistical Analysis}

Scores were summarized as mean and standard deviations for each reader. Inter- 


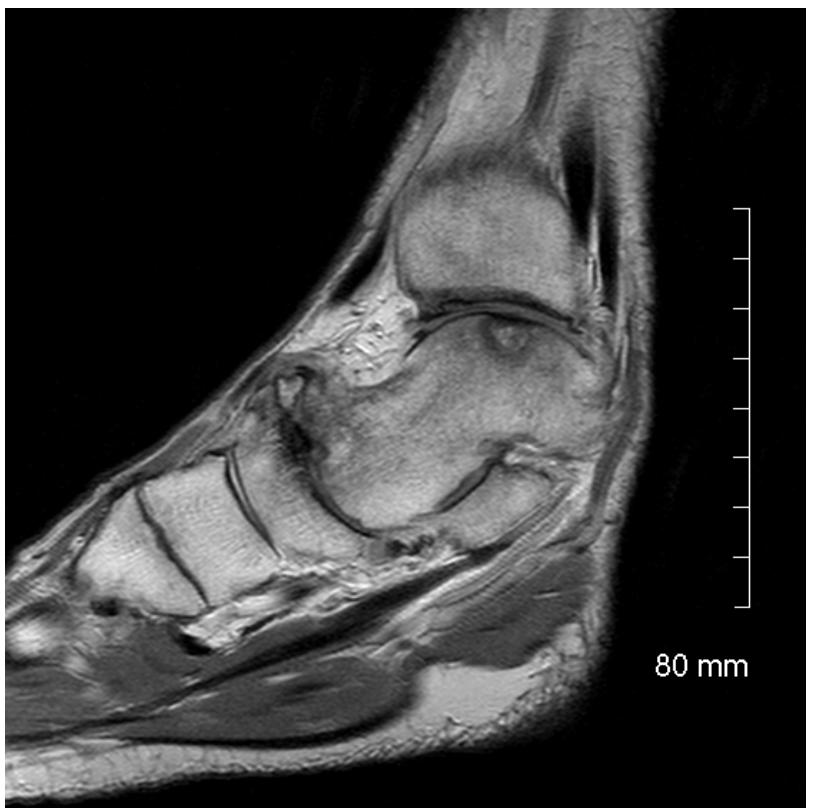

Figure 6. Sagittal PD weighted MR image showing osteophytes associated with the dorsal margin of the talo-navicluar joint.

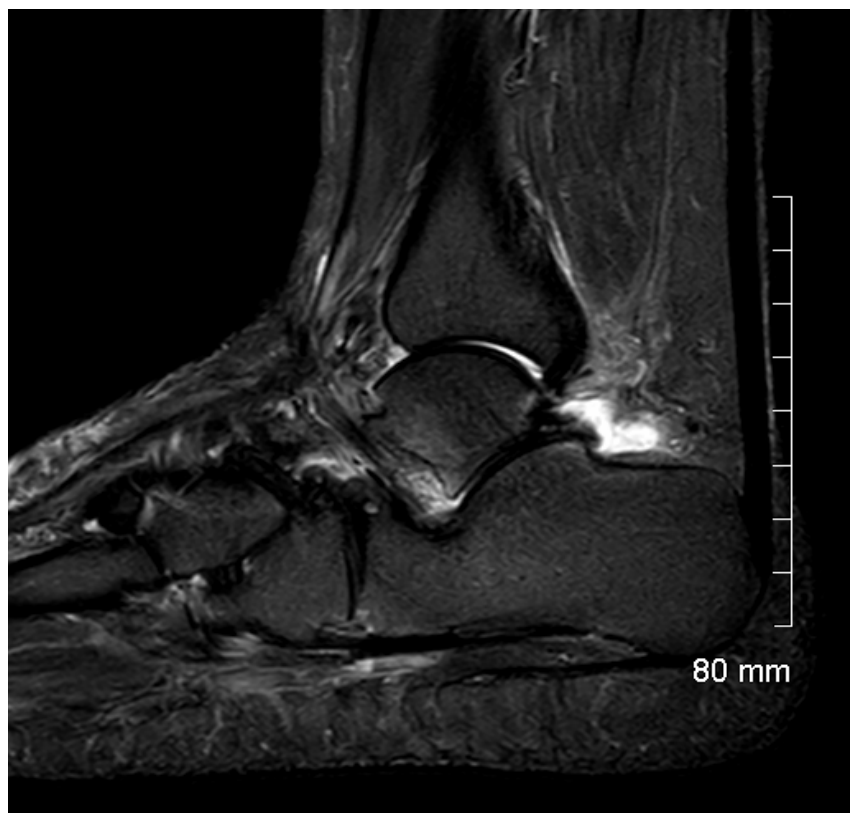

Figure 7. Sagittal STIR MR image showing synovitis at the posterior subtalar joint in the form of synovial stranding within a joint effusion.

and intra-observer agreement was based on the exact rating of each feature, not just the presence or absence of each feature of the scoring system. The data was treated as a continuous variable and therefore intraclass correlation coefficients (ICC) were the most appropriate test in order to calculate correlation. Cut-offs were used to grade inter- and intra-observer agreement. ICC values less than 0.40 were poor, between 0.40 and 0.59 were fair, values between 0.60 and 0.74 were good, and values between 0.75 and 1 excellent [9]. 


\subsection{Results}

The mean age of the cohort was 51 years (range 21 - 77) with $56 \%$ of patients being female. Indications for the MRI studies included assessment of known subtalar arthritis $(\mathrm{n}=15)$ as well as a range of other indications in the group which were treated as normal controls $(n=15)$; these included: assessment of Achilles tendon pathology (5 patients), peroneal tendon problems (4 patients), general ankle pain (4 patients) and pes cavus (2 patients).

For the scan performed for known TCJ or TNJ OA the mean total score was 30.1. This was statistically significantly higher than the score of 14.7 for the control scans (Table 2), showing the ability of the scoring system to detect abnormal pathologies in the TCJ and the TNJ.

The overall inter-observer correlation for the TCJ between the three readers was excellent at 0.76 (Table 3). For the TNJ the total correlation was good at 0.67 (Table 3). The inter-observer ICC for the total score was 0.75 which showed excellent agreement between the three readers.

The total intra-observer correlation was excellent (Table 4) for the subatalar and talonavicular scores for the two readers (JF and $\mathrm{RH}$ ) who repeated their assessments on two separate occasions.

\section{Discussion}

The current work describes the development and reliability of an MRI scoring system for subtalar arthritis. We have demonstrated an excellent overall ICC for inter- and intra-observer reliability that is as good as any previous MRI systems used to score knee arthritis [10]. The score has also been shown to detect pathology in the TCJ \& TNJ compared with controls.

Table 2. Mean talonavicular joint (TNJ), talocalcaneal joint (TCJ) and total scores for 15 known TCJ/TNJ OA patients compared with 15 scans performed in the control group for other reasons.

\begin{tabular}{|c|c|c|c|c|c|}
\hline & Number & Mean TNJ score & Mean TCJ score & Mean total score & $\mathrm{P}$-value \\
\hline Known TCJ/TNJ OA & 15 & 8.1 & 22.0 & 30.1 & \\
\hline Other reason for MRI & 15 & 4.1 & 10.6 & 14.7 & $\mathrm{p}=0.0006$ \\
\hline
\end{tabular}

Table 3. Inter-observer reliability for scoring the talocalcaneal joint and talonavicular joint. The $\mathrm{p}$ value for all ICCs was less than 0.001 .

\begin{tabular}{|c|c|c|}
\hline Feature & Talocalcaneal Joint & Talonavicular Joint \\
\hline Cartilage & 0.83 (CI $0.68-0.92)$ & $0.62(\mathrm{CI} 0.43-0.71)$ \\
\hline Bone Marrow & $0.62(\mathrm{CI} 0.32-0.8)$ & $0.60($ CI $0.47-0.74)$ \\
\hline Cysts & 0.66 (CI $0.47-0.8)$ & $0.71(\mathrm{CI} 0.53-0.85)$ \\
\hline Osteophytes & $0.53(\mathrm{CI} 0.27-0.64)$ & $0.57(\mathrm{CI} 0.36-0.74)$ \\
\hline Synovitis & $0.56($ CI $0.36-0.78)$ & $0.55(\mathrm{CI} 0.34-0.63)$ \\
\hline Total & $0.76($ CI $0.54-0.84)$ & $0.67(\mathrm{CI} 0.41-0.77)$ \\
\hline
\end{tabular}


Table 4. Intra-observer reliability for the subtalar joint and talonavicular joint for two readers scoring 14 days apart using intra-class correlation (ICC). The p-value for all ICCs was less than 0.001 .

\begin{tabular}{|c|c|c|c|c|}
\hline \multirow{2}{*}{ Feature } & \multicolumn{2}{|c|}{ Talocalcaneal Joint } & \multicolumn{2}{|c|}{ Talonavicular Joint } \\
\hline & Reader 1 & Reader 2 & Reader 1 & Reader 2 \\
\hline Cartilage & 0.88 (CI $0.76-0.94)$ & $0.95(\mathrm{CI} 0.9-0.98)$ & $0.43($ CI $0.1-0.7)$ & $0.88(\mathrm{CI} 0.76-0.94)$ \\
\hline Bone Marrow & $0.85(\mathrm{CI} 0.68-0.93)$ & 0.97 (CI $0.94-0.99)$ & $0.4(\mathrm{CI} 0.04-0.64)$ & $0.82(\mathrm{CI} 0.66-0.91)$ \\
\hline Cysts & 0.6 (CI $0.23-0.75)$ & 0.87 (CI $0.74-0.94)$ & $0.62(\mathrm{CI} 0.33-0.8)$ & 0.74 (CI $0.61-0.89)$ \\
\hline Osteophytes & $0.6($ CI $0.3-0.8)$ & 0.9 (CI $0.8-0.95)$ & $0.73(\mathrm{CI} 0.51-0.9)$ & $0.89(\mathrm{CI} 0.78-0.95)$ \\
\hline Synovitis & 0.74 (CI $0.5-0.9)$ & $0.88($ CI $0.76-0.94)$ & $0.72($ CI $0.5-0.9)$ & 0.76 (CI $0.56-0.88)$ \\
\hline Total & 0.9 (CI $0.84-0.96)$ & 0.97 (CI $0.92-0.99)$ & $0.8($ CI $0.6-0.9)$ & $0.91(\mathrm{CI} 0.83-0.96)$ \\
\hline
\end{tabular}

Currently, the main system available to score arthritis is the radiographically based Kellgren and Lawrence Grading System (KLGS) [11]. Many studies have looked at the reliability of this system. Mayich et al. found the KLGS to have only moderate inter- and intra-observer reliability with no way of taking into account any inflammatory component to the arthritis that may be present [12]. A more recent study looking at KLGS with post-traumatic subtalar joints found the reliability to be good with correlation to clinical scores [13].

MRI has many advantages over plain radiographic analysis in this context. Specifically, MRI identifies soft-tissue changes, hyaline cartilage defects, synovitis and bone marrow lesions. These cannot be visualised on routine radiographs, and therefore are not taken into account when grading a disease process using plain radiographs alone.

Between the readers the overall inter-observer reliability for the TCJ was excellent (0.76) and for the TNJ joint was good (0.67) and certainly is improved on the KLGS which has only moderate inter- and intra-observer reliability with no way of taking into account any inflammatory component [12]. The increased variation when scoring the TNJ using MRI may be as a result of its size as small joint surfaces or close opposition making accurate evaluation more difficult. The inter-observer error for the recognition of changes in cartilage, bone marrow and cysts was good to excellent for both of the scored joints.

A limitation of this work is that we did not include the calcaneocuboid joint in analysis. Although some textbooks describe this joint as being part of the subtalar joint complex, the description of the talus and the way that it articulates with the surrounding bones is controversial in both the anatomic and clinical literature [14]. In general, the anatomic literature distinguishes between individual joints and separates out the talocalcaneal navicular joints (subtalar) from the TNJ and CCJ (Chopart articulation). The Chopart articulation is responsible for the motion between the hindfoot and the midfoot. The general consensus however is that the subtalar joint describes the motion of the talus with the calcaneum to which the navicular is directly connected hence we elected to focus 
on these joints for analysis in order to keep the scoring system as simple and hence as practicable as possible. In addition, some pilot analyses we performed suggested that the CCJ was less commonly involved and mainly in cases of severe trauma or longstanding inflammatory arthropathy with hindfoot collapse and was invariably obvious on radiographic assessment. Given that this system was being developed with the aim of supporting a clinical study to compare the outcomes of ankle replacement and ankle fusion, where, in the absence of major deformity, adjacent joint arthritis appears to mainly affect the TCJ and TNJ, it was considered important to ensure a methodology that was not too cumbersome, and hence that could have utility for clinical practice or clinical trials. Further work however is necessary to document the CCJ pathology appearances on MRI. A further limitation of our work is that we have not attempted to correlate MRI scores to clinical outcomes but this will become the focus of future work.

\section{Conclusion}

We have developed and validated a novel MRI scoring system for subtalar joint arthritis that is easy to perform and demonstrates excellent reliability and believe would be a useful tool for clinical trials and other studies to diagnose and monitor disease progression.

\section{Acknowledgements}

We would like to acknowledge the TARVA study group and the Foot \& Ankle Unit at the Royal National Orthopaedic Hospital for its leadership on this important study as well as the radiology department at the Royal National Orthopaedic Hospital for use of its systems.

\section{Conflicts of Interest}

The authors declare no conflicts of interest regarding the publication of this paper.

\section{References}

[1] Peterfy, C.G., Guermazi, A., Zaim, S., et al. (2004) Whole-Organ Magnetic Resonance Imaging Score (WORMS) of the Knee in Osteoarthritis. Osteoarthritis andCartilage, 12, 177-190. https://doi.org/10.1016/j.joca.2003.11.003

[2] Lee, S., Nardo, L., Kumar, D., et al. (2015) Scoring Hip Osteoarthritis with MRI (SHOMRI): A Whole Joint Osteoarthritis Evaluation System. Journal of Magnetic Resonance Imaging, 41, 1549-1557. https://doi.org/10.1002/jmri.24722

[3] Goldberg, A.J., Zaidi, R., Thomson, C., et al. (2016) Total Ankle Replacement versus Arthrodesis (TARVA): Protocol for a Multicentre Randomised Controlled Trial BMJ Open, 6, e012716. https://doi.org/10.1136/bmjopen-2016-012716

[4] Jorm, A.F. (2015) Using the Delphi Expert Consensus Method in Mental Health Research. Australian \& New Zealand Journal of Psychiatry, 49, 887-897. https://doi.org/10.1177/0004867415600891 
[5] Barr, C., Bauer, J.S., Malfair, D., et al. (2007) MR Imaging of the Ankle at 3 Tesla and 1.5 Tesla: Protocol Optimization and Application to Cartilage, Ligament and Tendon Pathology in Cadaver Specimens. European Radiology, 17, 1518-1528. https://doi.org/10.1007/s00330-006-0446-4

[6] Demehri, S., Hafezi-Nejad, N. and Carrino, J.A. (2015) Conventional and Novel Imaging Modalities in Osteoarthritis: Current State of the Evidence. Current Opinion in Rheumatology, 27, 295-303. https://doi.org/10.1097/BOR.0000000000000163

[7] Guermazi, A., Niu, J., Hayashi, D., et al. (2012) Prevalence of Abnormalities in Knees Detected by MRI in Adults without Knee Osteoarthritis: Population Based Observational Study (Framingham Osteoarthritis Study). BMJ, 345, e5339-e5339. https://doi.org/10.1136/bmj.e5339

[8] Wenham, C.Y.J., Grainger, A.J. and Conaghan, P.G. (2014) The Role of Imaging Modalities in the Diagnosis, Differential Diagnosis and Clinical Assessment of Peripheral Joint Osteoarthritis. Osteoarthritis Cartilage, 22, 1692-1702. https://doi.org/10.1016/j.joca.2014.06.005

[9] Cicchetti, D.V. (1994) Guidelines, Criteria, and Rules of Thumb for Evaluating Normed and Standardized Assessment Instruments in Psychology.

[10] Hunter, D.J., Lo, G.H., Gale, D., Grainger, A.J., Guermazi, A. and Conaghan, P.G. (2008) The Reliability of a New Scoring System for Knee Osteoarthritis MRI and the Validity of Bone Marrow Lesion Assessment: BLOKS (Boston Leeds Osteoarthritis Knee Score). Annals of the Rheumatic Diseases, 67, 206-211. https://doi.org/10.1136/ard.2006.066183

[11] Kellgren, J.H. and Lawrence, J.S. (1957) Radiological Assessment of Osteo-Arthrosis. Annals of the Rheumatic Diseases, 16, 494-502. https://doi.org/10.1136/ard.16.4.494

[12] Mayich, D.J., Pinsker, E., Mayich, M.S., Mak, W. and Daniels, T.R. (2013) An Analysis of the Use of the Kellgren and Lawrence Grading System to Evaluate Peritalar Arthritis Following Total Ankle Arthroplasty. Foot \& Ankle International, 34, 1508-1515. https://doi.org/10.1177/1071100713495379

[13] Holzer, N., Salvo, D., Marijnissen, A.C.A., et al. (2015) Radiographic Evaluation of Posttraumatic Osteoarthritis of the Ankle: The Kellgren-Lawrence Scale Is Reliable and Correlates with Clinical Symptoms. Osteoarthritis Cartilage, 23, 363-369. https://doi.org/10.1016/j.joca.2014.11.010

[14] Bartoníček, J., Rammelt, S. and Naňka, O. (2018) Anatomy of the Subtalar Joint. Foot and Ankle Clinics, 23, 315-340. https://doi.org/10.1016/j.fcl.2018.04.001 